\title{
OPEN The use of pulse pressure variation for predicting impairment of microcirculatory blood flow
}

\author{
Christoph R. Behem ${ }^{1 凶}$, Michael F. Graessler ${ }^{1}$, Till Friedheim ${ }^{1}$, Rahel Kluttig ${ }^{1}$, \\ Hans O. Pinnschmidt ${ }^{2}$, Anna Duprée ${ }^{3}$, E. Sebastian Debus ${ }^{4}$, Daniel A. Reuter ${ }^{5}$, \\ Sabine H. Wipper ${ }^{6}$ \& Constantin J. C. Trepte ${ }^{1}$
}

Dynamic parameters of preload have been widely recommended to guide fluid therapy based on the principle of fluid responsiveness and with regard to cardiac output. An equally important aspect is however to also avoid volume-overload. This accounts particularly when capillary leakage is present and volume-overload will promote impairment of microcirculatory blood flow. The aim of this study was to evaluate, whether an impairment of intestinal microcirculation caused by volume-load potentially can be predicted using pulse pressure variation in an experimental model of ischemia/ reperfusion injury. The study was designed as a prospective explorative large animal pilot study. The study was performed in 8 anesthetized domestic pigs (German landrace). Ischemia/reperfusion was induced during aortic surgery. $6 \mathrm{~h}$ after ischemia/reperfusion-injury measurements were performed during 4 consecutive volume-loading-steps, each consisting of $6 \mathrm{ml} \mathrm{kg}^{-1}$ bodyweight ${ }^{-1}$. Mean microcirculatory blood flow (mean Flux) of the ileum was measured using direct laser-specklecontrast-imaging. Receiver operating characteristic analysis was performed to determine the ability of pulse pressure variation to predict a decrease in microcirculation. A reduction of $\geq 10 \%$ mean Flux was considered a relevant decrease. After ischemia-reperfusion, volume-loading-steps led to a significant increase of cardiac output as well as mean arterial pressure, while pulse pressure variation and mean Flux were significantly reduced (Pairwise comparison ischemia/reperfusion-injury vs. volume loading step no. 4): cardiac output $\left(\mathrm{I} \mathrm{min}^{-1}\right) 1.68(1.02-2.35)$ versus $2.84(2.15-3.53), p=0.002$, mean arterial pressure $(\mathrm{mmHg}) 29.89(21.65-38.12)$ versus $52.34(43.55-61.14), p<0.001$, pulse pressure variation (\%) 24.84 (17.45-32.22) versus 9.59 (1.68-17.49), $p=0.004$, mean Flux (p.u.) 414.95 (295.18-534.72) versus 327.21 (206.95-447.48), $p=0.006$. Receiver operating characteristic analysis revealed an area under the curve of $0.88(\mathrm{Cl} 95 \% 0.73-1.00 ; p$ value $<0.001)$ for pulse pressure variation for predicting a decrease of microcirculatory blood flow. The results of our study show that pulse pressure variation does have the potential to predict decreases of intestinal microcirculatory blood flow due to volumeload after ischemia/reperfusion-injury. This should encourage further translational research and might help to prevent microcirculatory impairment due to excessive fluid resuscitation and to guide fluid therapy in the future.

Abbreviation

PPV Pulse pressure variation

${ }^{1}$ Department of Anesthesiology, Center of Anesthesiology and Intensive Care Medicine, University Medical Center Hamburg-Eppendorf, Martinistraße 52, 20246 Hamburg, Germany. ${ }^{2}$ Department of Medical Biometry and Epidemiology, University Medical Center Hamburg-Eppendorf, Hamburg, Germany. ${ }^{3}$ Department of Visceraland Thoracic Surgery, Center of Operative Medicine, University Medical Center Hamburg-Eppendorf, Hamburg, Germany. ${ }^{4}$ Department of Vascular Medicine, University Heart and Vascular Center Hamburg GmbH (UHZ), Hamburg, Germany. ${ }^{5}$ Department of Anesthesiology and Intensive Care Medicine, Rostock University Medical Center, Rostock, Germany. ${ }^{6}$ University Department for Vascular Surgery, Department of Operative Medicine, Medical University of Innsbruck, Innsbruck, Austria. ${ }^{\circledR}$ email: c.behem@uke.de 


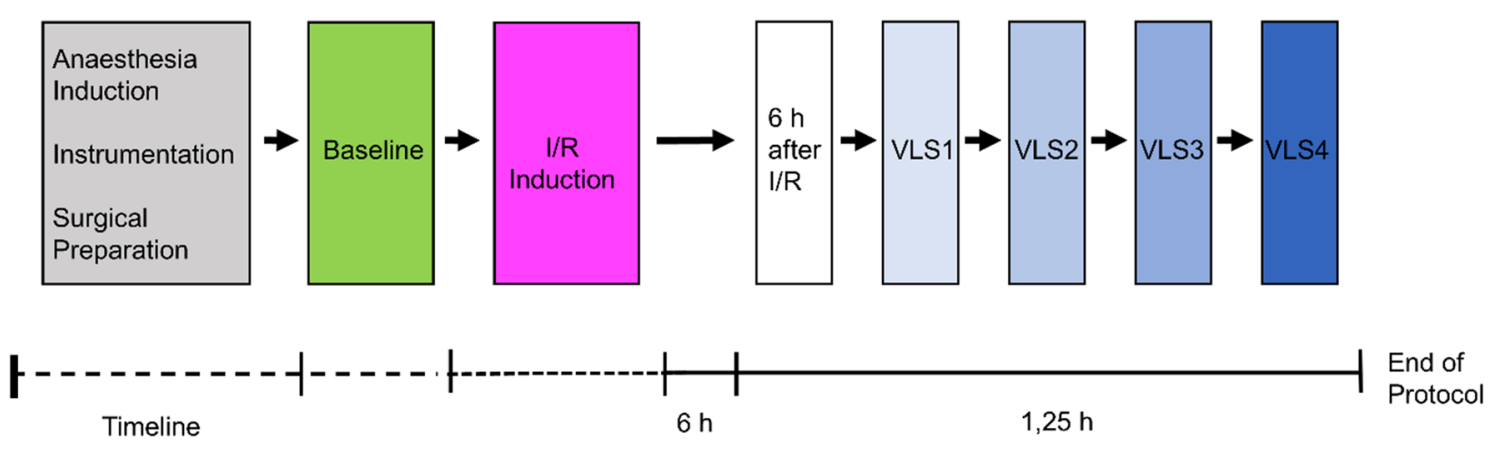

Figure 1. Experimental protocol. After completion of anesthesia induction, instrumentation and surgical preparations, baseline measurements were performed. Thereafter, ischemia/reperfusion (I/R) was induced. $6 \mathrm{~h}$ after ischemia/reperfusion induction, measurements were repeated and 4 consecutive volume-loading-steps (VLS) performed.

Dynamic parameters of preload like pulse pressure variation (PPV) were shown to be capable of predicting macrohemodynamic responses to fluid administration ${ }^{1,2}$. This led to the recommendation to use these parameters for guiding fluid therapy in critically-ill patients both perioperatively as well as in intensive care unit settings ${ }^{3-5}$. The rationale for optimizing macrohemodynamics using PPV aims to increase cardiac output, assuming that this would result in improved tissue perfusion. However, coherence between macro- and microcirculation is frequently lost emphasizing the need for direct microcirculatory evaluation ${ }^{6-8}$.

An important aspect in guiding fluid therapy is that both hypovolemia but also particularly volume-overload have to be avoided ${ }^{9,10}$. In an earlier study in an experimental model of systemic inflammation we were able to demonstrate that already full utilization of preload reserve will result in volume-overload accompanied by impairments of microcirculatory blood flow and endothelial function ${ }^{11}$. This is clinically important since the occurrence of microcirculatory disturbances is a major cause for the development of multiple-organ failure and accounts for increased mortality ${ }^{12-14}$. Tissue perfusion and thus oxygenation depend on the perfusion of the microvasculature ${ }^{6}$. The effects of fluid administration on microcirculatory blood flow so far have not been sufficiently addressed. While some investigators have revealed promising results, other studies have failed to show an improvement of microcirculation due to fluid therapy, even if therapy was guided in accordance with macrohemodynamic goals ${ }^{15-17}$.

With regard to PPV, certain values can discriminate fluid-responders from non-responders, while also a zone of intermediate values with limited discriminatory power has been identified ${ }^{18,19}$. Beneath this zone an increase of stroke volume is rather unlikely, which on the other hand makes it all the more likely that fluid administration will promote volume-overload and consequently also a decrease of microcirculatory blood flow. This might be of particular interest in conditions of disturbed endothelial function and increased capillary leakage.

Therefore, we hypothesized that the underlying physiological principles of PPV could also be used to identify whether fluid administration results in a decrease of microcirculatory blood flow. We used a porcine model of ischemia/reperfusion injury during experimental aortic surgery as a model for a condition with microcirculatory disturbances, endothelial dysfunction and increased capillary leakage ${ }^{20,21}$. The aim of this study was to evaluate whether PPV does have the potential to predict an impairment of intestinal microcirculatory blood flow due to volume-load in our experimental setting.

\section{Results}

Study population. 8 animals were studied. All animals survived until completion of protocol. No adverse events (e.g. severe bleeding, cardiac arrest) occurred. Mean body weight was 78.1 kg (95\% CI 75.9-80.3). The study protocol is given in Fig. 1 .

Hemodynamic parameters. Sample pictures of intestinal mean Flux are given in Fig. 2. Baseline values prior ischemia/reperfusion as well as changes of micro- and macrohemodynamic parameters throughout the experimental protocol after ischemia/reperfusion are shown in Fig. 3.

Prediction of intestinal microcirculatory blood flow decrease in ischemia/reperfusion. In each animal 4 volume-loading-steps were performed resulting in a total of 32 volume-loading-steps. 3 volume-loading-steps had to be excluded due to artefacts in microcirculatory measurements. Of the remaining 29 volumeloading-steps, 3 PPV values had to be excluded due to cardiac arrhythmias. Thus, in total 26 volume-loadingsteps were analyzed. There were 11 positive and 15 negative states. In detail for predicting decrease of intestinal microcirculatory blood flow in ischemia/reperfusion central PPV presented with an AUC of 0.88 (95\% CI 0.74$1.00 ; p<0.001)$. The receiver operating characteristic curve is presented in Fig. 4.

According to the receiver operating characteristic analysis in this experimental model of ischemia/reperfusion in pigs, the following cutoff-values for the prediction of microcirculatory decreases can be proposed: PPV $\leq 7.7 \%$ (Youden Index 0.75 ; sensitivity $81.82 \%$, specificity $93.33 \%$, positive predictive value 0.90 , negative predictive value 0.88 ). 


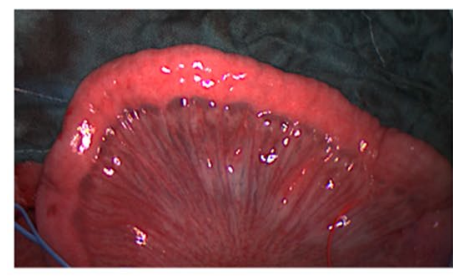

Colour photo



VLS2

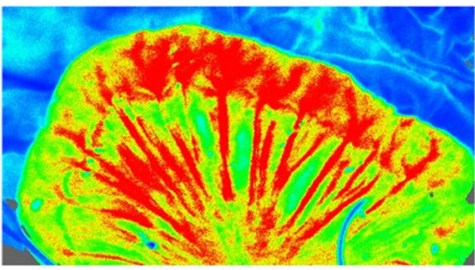

$\mathrm{I} / \mathrm{R}$

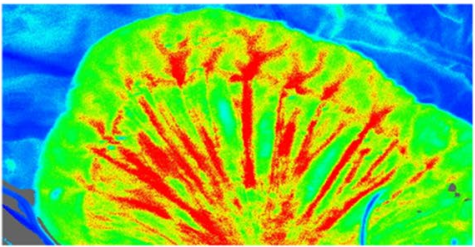

VLS3

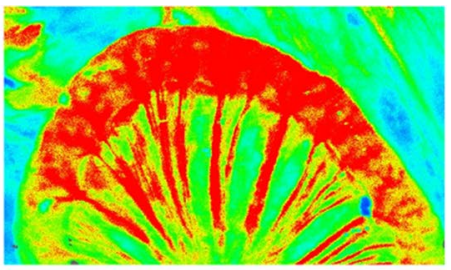

VLS1

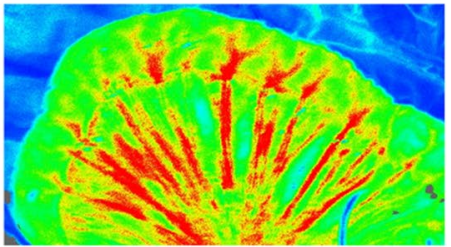

VLS4



Figure 2. Sample picture of small intestine and color-coded examples of Laser-Speckle-Contrast-Imaging. Sample picture of exposed small intestine (ileum) and color-coded examples of Laser-Speckle-Contrast-Imaging derived mean intestinal microcirculatory blood flow (mFlux) at all points of measurement in an exemplary animal. $\mathrm{I} / \mathrm{R}=$ ischemia/reperfusion; $\mathrm{VLS}=$ volume-loading-step.
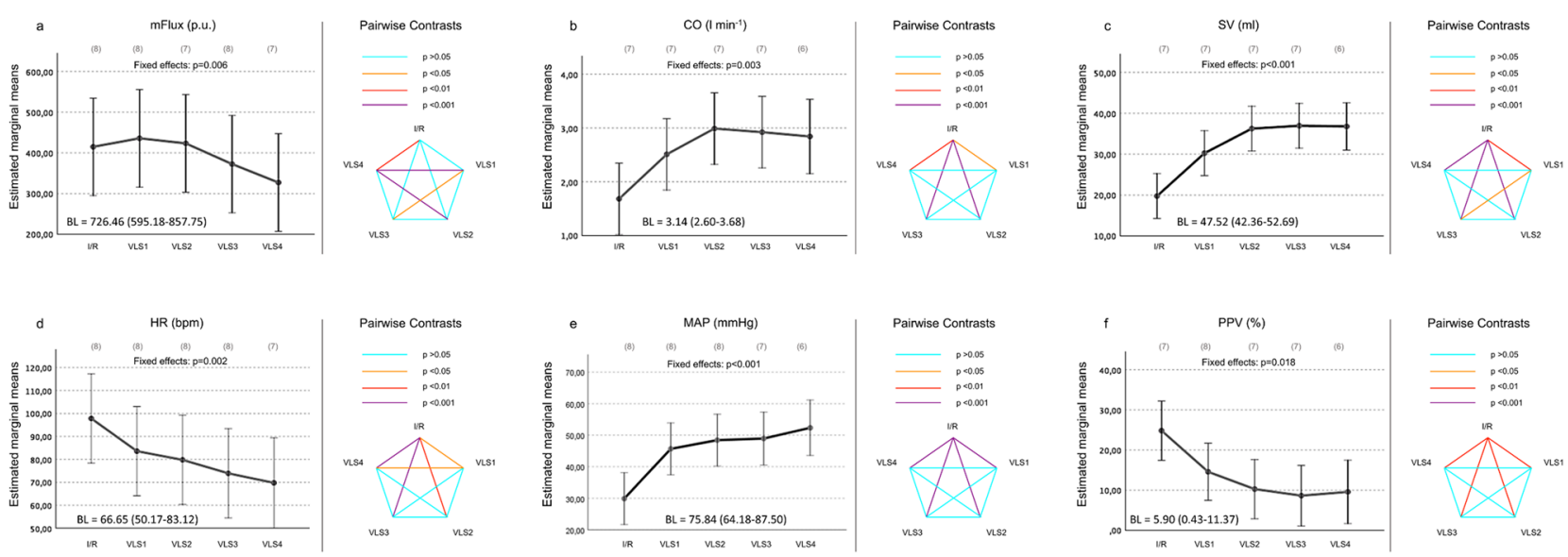

Figure 3. Changes of hemodynamic parameters throughout the experimental protocol. Changes throughout the experimental protocol of (a) mean microcirculatory blood flow (mFlux), (b) cardiac output (CO), (c) stroke volume (SV), (d) heart rate (HR), (e) mean arterial pressure (MAP) and (f) central pulse-pressure-variation (PPV). Data are presented as baseline adjusted estimated marginal means with $95 \%$ confidence intervals (left side). Results of pairwise comparisons are illustrated with color-coding of significance (right side). Points of measurements are $6 \mathrm{~h}$ after ischemia/reperfusion (I/R), volume loading steps 1-4 $6 \mathrm{~h}$ after ischemia/reperfusion (VLS1-4). Number of valid values for each point of measurement are given in brackets. Baseline values (BL) prior ischemia/reperfusion are given in addition.

In addition, receiver operating characteristics analysis was performed using peripheral PPV. Peripheral PPV presented with an AUC of 0.83 (95\% CI 0.66 to 1.00; $p=0.005)$ for prediction of intestinal microcirculatory blood flow decline with a cutoff value $\leq 8.0$ (Youden Index 0.59 , sensitivity $72.73 \%$, specificity $86.67 \%$, positive predictive value 0.80 , negative predictive value 0.81 ). Details on receiver operating characteristics analysis are given as additional table $2 \mathrm{~h}$ in the additional file.

\section{Discussion}

Our study demonstrates that PPV potentially could be used to predict decreases of intestinal microcirculatory blood flow due to excessive volume-load in an experimental setting of ischemia/reperfusion. Full utilization of preload reserve as reflected in low values for PPV aggravated impairment of intestinal microcirculatory flow. 


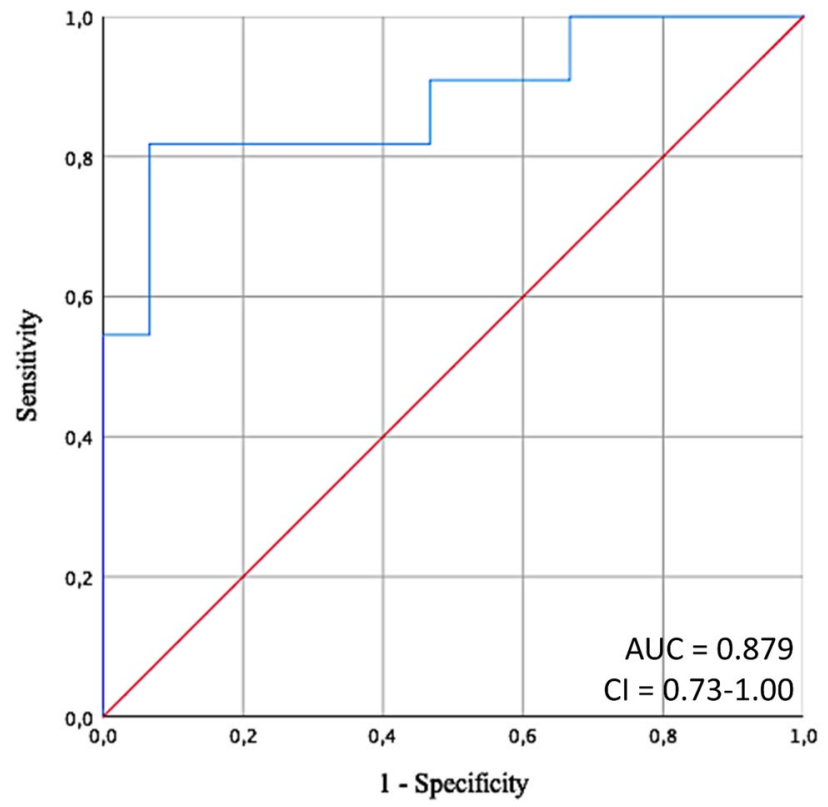

Figure 4. Receiver operating characteristic curve. Receiver operating characteristic curve to determine the ability of central pulse pressure variation to predict $\mathrm{a} \geq 10 \%$ decrease of small intestinal microcirculation after ischemia/reperfusion. Direction of the test was set to decreasing values of pulse pressure variation. AUC=area under the curve, $\mathrm{CI}=95 \%$ confidence interval.

This suggests that dynamic indicators of preload may also be suitable to prevent microcirculatory deterioration when guiding fluid therapy in the future.

Over-infusion can bring about aggravated tissue edema leading to a deterioration of microcirculatory blood flow, while microcirculatory deterioration will result in oxygen deficits, multiple-organ failure and is associated with increased mortality ${ }^{12-14}$. Existing studies focusing on the effects of fluid administration on microcirculation often have shown a failure to improve microcirculation during fluid therapy and a saturation of the volume effect has been already described ${ }^{15,16,22}$. Therefore, identifying potential parameters that would enable prediction of microcirculatory behavior is of highest interest. Dynamic preload parameters such as pulse pressure variation can be used to assess fluid responsiveness ${ }^{3,23-26}$. Moreover, they may be useful to prevent volume-overload ${ }^{19,27,28}$. However, studies considering microcirculatory effects are scarce.

Loss of hemodynamic coherence has been described for different scenarios including perioperative medicine ${ }^{6,7,29-31}$. Microcirculatory alterations have a huge impact on prognosis and outcome not only in sepsis and heart failure, but also in surgical patients ${ }^{29,31,32}$. Moreover, anesthesia itself affects microcirculation ${ }^{31,33,34}$. Ischemia/reperfusion occurs in several medical conditions and leads to microcirculatory deterioration, accounts for the development of multiple-organ failure and is associated with increased mortality ${ }^{12,13,20,21,35,36}$. Alterations in microcirculatory blood flow have been shown to correlate with clinical outcome after liver, kidney and pancreas transplanation ${ }^{31,37-39}$. Ischemia/reperfusion induced by aortic cross clamping has been shown to influence microcirculation ${ }^{31}$. Loss of hemodynamic coherence with impairments of microcirculation as well as endothelial dysfunction has been described and microcirculation correlated with outcome following aortic cross clamping and vascular surgery $\mathrm{y}^{8,40-42}$. In contrast, protection of splanchnic microcirculation with use of colloids has been shown to reduce complications in abdominal aortic surgery ${ }^{43}$. Intestinal microcirculatory alterations have also been shown to be associated with anastomotic leakage ${ }^{31}$. The small intestine is particularly susceptible to ischemia/reperfusion-injury resulting in intestinal-barrier dysfunction, bacterial translocation, systemic inflammation and multiple-organ failure ${ }^{21,44,45}$. Microcirculatory deteriorations are important determinants for manifestation of intestinal ischemia/reperfusion injury and have major prognostic impact ${ }^{46}$. The results of this study has revealed a promising new field of application for PPV as being capable of predicting impairments of intestinal microcirculatory blood flow in ischemia/reperfusion. Since effects of fluids on microvascular perfusion are associated with outcome $\mathrm{e}^{32}$, use of PPV for prevention of small intestinal microcirculatory deterioration during fluid administration does have the potential to improve outcome.

In our experimental setting of ischemia/reperfusion PPV presented with a good AUC for predicting decline of microcirculatory blood flow due to volume-load. The cut-off value $\leq 7.7 \%$ in our series would be interpreted as being in the gray zone or to indicate and correspond to non-responders with regard to macrocirculatory responses to a fluid challenge. Cannesson and colleagues identified PPV-values between 9 and 13\% as being inconclusive while Biais and others also showed that PPV-values between 4 and $17 \%$ failed to accurately predict fluid responsiveness ${ }^{18,19}$. Biais and others have reported, that this "grey zone" is already associated with the potential risk of over-infusion ${ }^{19}$. 
Since most of clinical studies use PPV derived by femoral or radial artery blood pressure measurements ${ }^{18,47}$, peripheral PPV was assessed in addition. Although the AUC for peripheral PPV was lower, which may be explained be our experimental model using an aortic graft potentially affecting PPV distal of the graft ${ }^{48}$, it still presented with an acceptable AUC. Therefore, our results suggest that either central or peripheral PPV can be used for prediction of microcirculatory decline, which would facilitate translation to a clinical scenario.

Our results suggest that utilization of preload reserve after ischemia/reperfusion should be performed with particular caution avoiding fluid administration if stroke volume increase is uncertain as indicated by PPV-values below or inside the grey zone. In this regard, a prospective randomized clinical trial demonstrated an improved outcome using a fluid sparing regimen and limiting fluid boluses to presence of hypotension, low cardiac output and high stroke volume variation ${ }^{27}$. Biais and colleagues already tried to take into account the patients' clinical condition according to $\mathrm{PaO}_{2} / \mathrm{FiO}_{2}$ ratios when trying to define optimal threshold values for PPV aiming to minimize the risk of volume-overload ${ }^{19}$. Volume-overload has clearly been shown to be associated with worsened outcome ${ }^{10,49,50}$. However, in patients at increased risk for complications during major abdominal surgery, a restrictive fluid regimen was not associated with improved survival and was associated with a higher rate of acute kidney injury ${ }^{51}$. Nevertheless, a fluid regimen tailored to microcirculatory demands may improve outcome, even if it is guided by macrohemodynamic parameters. An association between elevated central venous pressure and microcirculatory deterioration has already been described for septic patients ${ }^{52}$. Moreover, goal-directed therapy based on macrohemodynamic parameters has been shown to improve microcirculation in experimental pancreatitis, major surgery as well as abdominal surgery with important implications for outcome, and even in aortic surgery, prevention of microcirculatory decline could recently been shown by use of a goal directed hemodynamic management ${ }^{53-56}$. While hemodynamic coherence is frequently lost in various conditions ${ }^{6,31}$, association between pulse pressure variation and intestinal microcirculation may still exist. A potential association between pulse pressure variation and microcirculation may also be suggested by previous studies. While a loss of hemodynamic coherence was found for septic patients reflected by a dissociation between sublingual microcirculation and cardiac index as well as mean arterial pressure, this was only found in the late phase of sepsis. In the early phase of sepsis there was no dissociation between macro- and microcirculatory parameters. Most interesting, this was accompanied by a significant reduction of pulse pressure variation, while in the late phase, no significant change of pulse pressure variation was seen ${ }^{16}$. Moreover, an improvement of microcirculatory parameters following passive leg raise as well as fluid administration has already been shown for preload dependent septic patients ${ }^{15}$. In our project, there was a dissociation between the systemic variables cardiac output, stroke volume and mean arterial pressure, which has previously been discussed ${ }^{57}$. Nevertheless, pulse pressure variation predicted microcirculatory decline. The results of our study are also in line with the results of a recent study conducted in patients undergoing major surgery by Bouattour et al.. In this clinical study, the authors could demonstrate that fluid administration in conditions of preload dependency as identified by a pulse pressure variation $>13 \%$ led to an improved microcirculation as assessed with sublingual sidestream darkfield imaging ${ }^{47}$. These results underline the usefulness of PPV to guide treatment of microcirculation. Based on the ability of PPV to predict microcirculatory decline due to fluid administration as shown in this study, we propose that in ischemia/reperfusion conditions, a PPV-based fluid management may help to protect intestinal microcirculation.

Our study has certain limitations. First, we limited the number of animals in this hypothesis generating pilot study. Sensible a priori input data to be used for sample size and power calculations were not available, hence, we chose a sample size that appeared feasible with respect to size of the study protocol and in accordance to ARRIVE and FELASA guidelines as well as 3R-principles reducing animal numbers to a reasonable sample size while being able to address the scientific topic properly ${ }^{58}$. Moreover, the number of animals as well as volumeloading-steps is comparable with existing studies ${ }^{59-64}$. In addition, the number of volume-loading-steps is also comparable to a number of clinical studies ${ }^{65-72}$. As this was an pilot study for generating hypotheses rather than confirmatory test pre-specified hypotheses, alpha error adjustment for multiple testing was not done. In addition, we did not include a control group. However, we assumed that the risk for microcirculatory deterioration due to fluid therapy would be low in healthy conditions and that there would be to few positive states to reliably assess the ability of PPV for prediction of microcirculatory decline in a control group. Another limitation would be that duration of vessel ischemia was not intended as standardized ischemia times but was dependent on the surgical techniques used. Although ischemic times were different between animals, the variation of vessel ischemia would reflect the clinical situation of open thoraco-abdominal aortic repair, especially since surgical procedures were performed by experienced vascular surgeons and the advantage of the study would therefore be the comparability to the clinical scenario ${ }^{73-75}$. Moreover, besides predicting decreases of intestinal microcirculatory blood flow caused by volume-overload, the clinical impact of this microcirculatory decline was not further assessed in our study. However, this has already been shown by previous publications ${ }^{31,32}$. Choice of anesthesia can also influence microcirculation ${ }^{31}$. Therefore, we have used a standardized approach with identical anesthesia doses and identical ventilation settings. Moreover, in a recent study on the effects of total intravenous vs. balanced anesthesia, use of a goal-directed therapy prevented deterioration of microcirculatory perfusion as well as oxygenation for both groups ${ }^{56}$. Therefore, our results may also be translated to patients receiving balanced anesthesia. Different effects of vasoactive drugs on microcirculation have been described ${ }^{31,76}$. Although all vasopressors used during surgical phases have been terminated for at least $30 \mathrm{~min}$ prior measurements, we cannot rule out residual effects of vasopressors even if they are very much unlikely after $30 \mathrm{~min}$ of cessation. While the choice of fluids does not influence the predictive value of $\mathrm{PPV}^{26}$, colloids may be different from crystalloids in regard to microcirculation. While some authors found no benefits or even detrimental effects for colloids, others have found beneficial effects in regard to microcirculation ${ }^{27,31,77-89}$. Choice of fluids was based on the experience from previous studies showing pronounced effects of hydroxyethyl-starch colloids on macro- as well as microcirculation in our porcine models ${ }^{11,53,90-94}$. Moreover, detrimental effects on microcirculation have been described for crystalloids as well ${ }^{95-98}$. Nevertheless, future studies are needed to test the ablity of pulse pressure 
varation to predict microcirculatory decline during fluid administration with crystalloids as well. In regard to laser speckle contrast imaging it should be noted that capillaries have a diameter of about $7 \mu \mathrm{m}^{99,100}$, while laser speckle contrast imaging technique has a maximum resolution of about $10 \mu \mathrm{m}$, so it is barely possible to directly visualize capillaries with laser speckle contrast imaging. However, it is possible to detect perfusion in a capillary bed even when capillaries cannot be identified individually ${ }^{100}$. Nevertheless, the limitation of the used method for microcirculatory evaluation should be regarded. Future studies should also investigate the usefulness of dynamic preload parameters for maintenance and optimization of microcirculation in different clinical settings and other organs.

In conclusion, the results of our study show that PPV does have the potential to predict decreases of intestinal microcirculatory blood flow caused by volume-load in an experimental setting of ischemia/reperfusion. Full utilization of preload as reflected in low values of PPV resulted in impaired microcirculatory blood flow. This should encourage further translational research and might help to prevent microcirculatory impairment due to excessive fluid resuscitation and to guide fluid therapy in the future.

\section{Methods}

Study design. The study was conducted as a prospective explorative pilot study in 8 anesthetized domestic pigs (German landrace) both female and male using animals weighing approximately $75-80 \mathrm{~kg}$. This study was performed in combination with a feasibility study for a new hybrid-graft implantation in accordance to ARRIVE and FELASA guidelines and 3Rs-principles reducing animal number. The animals received care in compliance with the 'Guide for the Care and Use of Laboratory Animals' (NIH publication No. 86-23, revised 2011) as well as FELASA guidelines and recommendations and experiments were carried out according to the ARRIVE guidelines $^{58,101}$. Please see the ARRIVE guidelines checklist as well as additional comments on the ARRIVE guidelines given in the additional file. Results on microcirculatory behavior have been previously reported and methods have been previously described ${ }^{57,102}$.

The study protocol is given in Fig. 1. This study was performed in coherence with current recommendations on the use of pulse pressure variation: All animals were mechanically ventilated with tidal volumes of $8 \mathrm{ml} \mathrm{kg}^{-1}$ total body weight, arrhythmic episodes were excluded and high respiratory rates avoided ${ }^{103,104}$. Ischemia/reperfusion was induced during aortic hybrid-graft implantation. Micro- and macrocirculation were measured at baseline prior ischemia/reperfusion and $6 \mathrm{~h}$ after ischemia/reperfusion induced by aortic hybrid-graft implantation. Thereafter 4 consecutive volume-loading-steps were performed followed by micro- and macrocirculatory measurements. Each volume-loading-step consisted of $6 \mathrm{ml} \mathrm{kg}^{-1}$ bodyweight ${ }^{-1}$ colloids (Voluven $6 \%$, Fresenius Kabi, Bad Homburg, Germany). Each volume-loading-step was performed during a time period of 5 min using pressurized infusions. After completion of each volume-loading-step, 5 min were allowed for equilibration. At least $30 \mathrm{~min}$ prior measurements, all vasopressors used during the surgical phase were terminated to exclude effects of these substances.

Assessment of microcirculation. Microcirculatory blood flow was directly assessed using laser speckle contrast imaging as previously described ${ }^{57}$. Laser speckle contrast imaging has been frequently used for monitoring of the microcirculation including intestinal microcirculation as well models of ischemia/reperfusion ${ }^{105-115}$. For use of laser speckle contrast imaging, tissues are illuminated with coherent laser light. The backscattered light from the tissue then forms a random interference pattern at the detector. This interference pattern is called speckle pattern ${ }^{106}$. Laser speckle contrast imaging is based on the laser Doppler method, however, it has a much higher spatial and temporal resolution with use of full field laser measurements. It allows imaging of large surface area, is contact-free and can measure perfusion in real time ${ }^{111}$. It can detect mean microcirculatory blood flow (mFlux) up to a tissue depth of $3 \mathrm{~mm}$. It is non-invasive and has good reproducibility ${ }^{115}$. The mean Flux (mFlux) is a dimensionless unit of microcirculatory blood flow, with the unit p.u. ${ }^{112}$. In detail, the speckle-laser (MoorFLPI-2, Moor Instruments, Axminster, UK) was positioned $25 \mathrm{~cm}$ above the intestinal segment using a target laser. For each measurement step microcirculatory blood flow was assessed for a $30 \mathrm{~s}$ period reducing variability due to respiration and organ movement $t^{57,111,116}$. A blinded investigator defined the region of interest off-line and calculated the mean Flux for the region of interest using a dedicated software (Moor FLPI-2 Review Software, v. 4.0, Moor Instruments, Axminster, UK) ${ }^{57}$.

Assessment of macrocirculation. Cardiac output and stroke volume were assessed invasively using a perivascular flow probe (Confidence PAU Flowprobe, chronic liner, 16 or $18 \mathrm{~mm}$, Transonic Systems Inc., Ithaca, NY, USA) that was fit around the descending aorta and connected to the adapted hardware (Perivascular Flow Module, Transonic Systems Inc., Ithaca, NY, USA). Invasive pressure catheters (Millar Micro-Tip pressure catheters, Houston, Texas, USA) for assessment of arterial pressure were directly inserted into the ascending aorta via the carotid artery for arterial pressure measurement and assessment of central PPV as well as in the femoral artery for assessment of peripheral PPV using 8 Fr. introducer sheaths. Macrohemodynamic measurements were recorded simultaneously with microcirculatory assessment for a period of $2 \mathrm{~min}$.

Data acquisition and processing. Invasive data were recorded by adapted hardware from ADInstruments (ADInstruments Bridge Amp and PowerLab, ADInstruments Ltd., Oxford, UK) and Transonic (Perivascular Flow Module, Transonic Systems Inc., Ithaca, NY, USA). Data analysis was performed offline using LabChart software (LabChart Pro, version 8, ADInstruments Ltd., Oxford, UK). Central and peripheral PPV were calculated using data from 10 respiratory cycles. The following formula was applied: PULSE PRESSURE VARIATION (\%) $=\frac{\text { (MaximumPulsePressure-MinimumPulsePressure) }}{(\text { MeanPulsePressure) }} * 100$ 
Animal care, anesthesia and surgical procedures. Animal care, anesthesia and surgical procedures have been previously described ${ }^{57,102}$. Animals were brought to animal care facilities at least 7 days prior experiments and were housed in accordance to animal welfare recommendations. Animals were given food and water ad libitum and health status was regularly assessed by the responsible veterinarian. A fasting time of $12 \mathrm{~h}$ was maintained prior to the experiments ${ }^{102}$. Anesthesia was performed intravenously using a combination of an opiod together with different hypnotic agents to secure deep anesthesia during the entire protocol as previously described ${ }^{57}$. For anesthesia induction all animals received intramuscular injections of ketamine $10 \mathrm{mg} \mathrm{kg}^{-1}$ bodyweight ${ }^{-1}$, azaperone $4 \mathrm{mg} \mathrm{kg}^{-1}$ bodyweight ${ }^{-1}$, atropine $0.01 \mathrm{mg} \mathrm{kg}^{-1}$ bodyweight $^{-1}$ and midazolam $10 \mathrm{mg}$ for premedication. Thereafter, all animals were surgically tracheotomized and the trachea was intubated via the tracheotomy ostium. Maintenance of anesthesia was performed by continuous infusion of propofol ( $4 \mathrm{mg} \mathrm{kg}^{-1}$ bodyweight $\left.{ }^{-1} \mathrm{~h}^{-1}\right)$, fentanyl $\left(10 \mu \mathrm{g} \mathrm{kg}^{-1}\right.$ bodyweight $\left.{ }^{-1} \mathrm{~h}^{-1}\right)$, midazolam $\left(0.3 \mathrm{mg} \mathrm{kg}^{-1}\right.$ bodyweight $\left.{ }^{-1} \mathrm{~h}^{-1}\right)$ and ketamine $\left(6 \mathrm{mg} \mathrm{kg}^{-1}\right.$ bodyweight $\left.{ }^{-1} \mathrm{~h}^{-1}\right)$. This combination provides adequate anesthesia as well as hemodynamic stability and has been successfully used in previous studies ${ }^{57,117,118}$. Adequacy of anesthesia was assessed by careful observation of vital signs and ventilation parameters as well as by absence of any movements during the entire protocol with special attendance to phases of surgical stimulus. Additional bolus doses of fentanyl $(50 \mu \mathrm{g})$ were given if there was any indication of pain or distress ${ }^{102}$. Pancuronium $\left(0.1 \mathrm{mg} \mathrm{kg}^{-1}\right.$ bodyweight $\left.^{-1}\right)$ was only given for tracheotomy. A volume-controlled ventilation was established using tidal volumes of $8 \mathrm{ml} \mathrm{kg}^{-1}$ bodyweight $^{-1}$ and a positive end-expiratory pressure of $8 \mathrm{cmH}_{2} \mathrm{O}$ and ventilator frequency was adjusted to maintain an endexpiratory carbon dioxide tension (etCO ${ }_{2}$ ) of 35-40 mmHg (Julian, Dräger Medical, Lübeck, Germany). All animals were placed in supine position on a warming blanket to prevent heat loss ${ }^{57,102}$. Surgical procedures have been previously described ${ }^{57,118}$ and are given in detail in the additional file. Duration of vessel ischemia was not intended as standardized ischemia times but was dependent on the surgical techniques used. Times of vessel ischemia are given as additional table 1 in the additional file. The entire experimental procedure and handling of the animals was supervised by the responsible veterinarian.

Euthanasia. After completion of the study protocol all animals were sacrificed during deep anesthesia by fast injection of $40 \mathrm{mmol}$ potassium chloride as previously described ${ }^{57,102}$.

Sample size calculation. As previously described, a priori input data to be used for sample size and power calculations were not available, hence, a sample size was chosen that appeared feasible with respect to size of the study protocol and in accordance to ARRIVE and FELASA guidelines and 3R-principles reducing animal numbers to a reasonable sample size while being able to address the scientific topic properly ${ }^{57}$.

Primary endpoint. Primary endpoint of this study was the ability of PPV to predict a mean microcirculatory flux decrease $\geq 10 \%$ following fluid administration.

Statistical analysis. The statistical plan was approved by all authors before start of the study. The dependent variables cardiac output, stroke volume, heart rate, mean arterial pressure, central PPV and mean Flux were subjected to general linear mixed model analyses, using the SPSS v. 24 routine GENLINMIXED for continuous data with an identity link function as previously described ${ }^{57}$. Models were specified with fixed effects for variable measurement point and random intercepts for animals. In addition, baseline values prior ischemia/reperfusion were included as fixed effects to the model as well to adjust for differences in baseline values between animals. Measurement points were considered as repeated measures within animals. Marginal means with 95\% CI were computed for all dependent variables at all measurement points, followed by multiple pairwise comparisons of measurement point means via least significant difference tests. The assessment of the ability of PPV to predict microcirculatory decline was performed by calculating receiver operating characteristic curves. The response to fluid administration was considered significant, if mean microcirculatory flux decreased by at least $10 \%$. Direction of the test was set to decreasing values of PPV, assuming that the likelihood of the state event increases with decreases in PPV. The value of the predictor variable resulting in a maximum Youden index ${ }^{119}$ was assumed to represent its ideal cutoff value. Statistical analyses were performed using the SPSS statistical software package 24 (IBM SPSS Statistics Inc., USA). Additional details on statistical analysis are given in the in the additional file as additional tables $2 \mathrm{a}-\mathrm{h}$. Variables are expressed as mean (95\% confidence interval, CI). Two-tailed $p$ values less than 0.05 were considered significant. In addition, results of general linear mixed model analyses that handled baseline values as part of the outcome and not as fixed effect covariate are given as additional table 3 , along with $p$ values of pairwise contrasts.

Ethics approval and consent to participate. The study was approved by the Governmental Commission on the Care and Use of Animals of the City of Hamburg (Reference-No. 101/15). The animals received care in compliance with the 'Guide for the Care and Use of Laboratory Animals' (NIH publication No. 86-23, revised 2011) as well as FELASA guidelines and recommendations and experiments were carried out according to the ARRIVE guidelines. Please see the ARRIVE guidelines checklist as well as additional comments on the ARRIVE guidelines given in the additional file.

\section{Data availability}

The datasets analysed during the current study are available from the corresponding author on reasonable request. Additional details on statistical analysis are given in the additional file. 
Received: 22 December 2020; Accepted: 12 April 2021

Published online: 28 April 2021

\section{References}

1. Pinsky, M. R. Functional hemodynamic monitoring. Crit. Care Clin. 31, 89-111. https://doi.org/10.1016/j.ccc.2014.08.005 (2015).

2. Monnet, X. \& Teboul, J. L. Assessment of fluid responsiveness: recent advances. Curr. Opin. Crit. Care 24, 190-195. https://doi. org/10.1097/MCC.0000000000000501 (2018).

3. Pinsky, M. R. Functional haemodynamic monitoring. Curr. Opin. Crit. Care 20, 288-293. https://doi.org/10.1097/MCC.00000 00000000090 (2014).

4. Cecconi, M. et al. Consensus on circulatory shock and hemodynamic monitoring. Task force of the European Society of Intensive Care Medicine. Intensive Care Med. 40, 1795-1815. https://doi.org/10.1007/s00134-014-3525-z (2014).

5. Rhodes, A. et al. Surviving sepsis campaign: international guidelines for management of sepsis and septic shock: 2016. Intensive Care Med. 43, 304-377. https://doi.org/10.1007/s00134-017-4683-6 (2017).

6. Ince, C. Hemodynamic coherence and the rationale for monitoring the microcirculation. Crit. care 19(Suppl 3), S8. https://doi. org $/ 10.1186 /$ cc14726 (2015).

7. De Backer, D., Ortiz, J. A. \& Salgado, D. Coupling microcirculation to systemic hemodynamics. Curr. Opin. Crit. Care 16, 250-254. https://doi.org/10.1097/MCC.0b013e3283383621 (2010).

8. Siegemund, M. et al. Aortic cross-clamping and reperfusion in pigs reduces microvascular oxygenation by altered systemic and regional blood flow distribution. Anesth. Analg. 111, 345-353. https://doi.org/10.1213/ANE.0b013e3181e4255f (2010).

9. Bellamy, M. C. Wet, dry or something else?. Br. J. Anaesth. 97, 755-757. https://doi.org/10.1093/bja/ael290 (2006).

10. Thacker, J. K., Mountford, W. K., Ernst, F. R., Krukas, M. R. \& Mythen, M. M. Perioperative fluid utilization variability and association with outcomes: considerations for enhanced recovery efforts in sample US surgical populations. Ann. Surg. 263, 502-510. https://doi.org/10.1097/SLA.0000000000001402 (2016).

11. Wodack, K. H. et al. Individualized early goal-directed therapy in systemic inflammation: is full utilization of preload reserve the optimal strategy?. Crit. Care Med. 42, e741-751. https://doi.org/10.1097/CCM.00000000000000657 (2014).

12. Sakr, Y., Dubois, M. J., De Backer, D., Creteur, J. \& Vincent, J. L. Persistent microcirculatory alterations are associated with organ failure and death in patients with septic shock. Crit. Care Med. 32, 1825-1831 (2004).

13. Trzeciak, S. et al. Early microcirculatory perfusion derangements in patients with severe sepsis and septic shock: relationship to hemodynamics, oxygen transport, and survival. Ann. Emerg. Med. 49, 88-98. https://doi.org/10.1016/j.annemergmed.2006. 08.021 (2007).

14. De Backer, D. et al. Microcirculatory alterations in patients with severe sepsis: impact of time of assessment and relationship with outcome. Crit. Care Med. 41, 791-799. https://doi.org/10.1097/CCM.0b013e3182742e8b (2013).

15. Pottecher, J. et al. Both passive leg raising and intravascular volume expansion improve sublingual microcirculatory perfusion in severe sepsis and septic shock patients. Intensive Care Med. 36, 1867-1874. https://doi.org/10.1007/s00134-010-1966-6 (2010).

16. Ospina-Tascon, G. et al. Effects of fluids on microvascular perfusion in patients with severe sepsis. Intensive Care Med. 36, 949-955. https://doi.org/10.1007/s00134-010-1843-3 (2010).

17. Edul, V. S. et al. Dissociation between sublingual and gut microcirculation in the response to a fluid challenge in postoperative patients with abdominal sepsis. Ann. Intensive Care 4, 39. https://doi.org/10.1186/s13613-014-0039-3 (2014).

18. Cannesson, M. et al. Assessing the diagnostic accuracy of pulse pressure variations for the prediction of fluid responsiveness: a "gray zone" approach. Anesthesiology 115, 231-241. https://doi.org/10.1097/ALN.0b013e318225b80a (2011).

19. Biais, M. et al. Clinical relevance of pulse pressure variations for predicting fluid responsiveness in mechanically ventilated intensive care unit patients: the grey zone approach. Crit. Care 18, 587. https://doi.org/10.1186/s13054-014-0587-9 (2014).

20. Granger, D. N. \& Kvietys, P. R. Reperfusion injury and reactive oxygen species: the evolution of a concept. Redox Biol. 6, 524-551. https://doi.org/10.1016/j.redox.2015.08.020 (2015).

21. Granger, D. N. Ischemia-reperfusion: mechanisms of microvascular dysfunction and the influence of risk factors for cardiovascular disease. Microcirculation 6, 167-178 (1999).

22. De Backer, D., Orbegozo Cortes, D., Donadello, K. \& Vincent, J. L. Pathophysiology of microcirculatory dysfunction and the pathogenesis of septic shock. Virulence 5, 73-79. https://doi.org/10.4161/viru.26482 (2014).

23. Pinsky, M. R. Heart lung interactions during mechanical ventilation. Curr. Opin. Crit. Care 18, 256-260. https://doi.org/10.1097/ MCC.0b013e3283532b73 (2012).

24. Marik, P. E., Monnet, X. \& Teboul, J. L. Hemodynamic parameters to guide fluid therapy. Ann. Intensive Care 1, 1. https://doi. org/10.1186/2110-5820-1-1 (2011).

25. Biais, M. et al. Changes in stroke volume induced by lung recruitment maneuver predict fluid responsiveness in mechanically ventilated patients in the operating room. Anesthesiology https://doi.org/10.1097/ALN.0000000000001459 (2016).

26. Yang, X. \& Du, B. Does pulse pressure variation predict fluid responsiveness in critically ill patients? A systematic review and meta-analysis. Crit. Care 18, 650. https://doi.org/10.1186/s13054-014-0650-6 (2014).

27. Luo, J. et al. Goal-directed fluid restriction during brain surgery: a prospective randomized controlled trial. Ann. Intensive Care 7, 16. https://doi.org/10.1186/s13613-017-0239-8 (2017).

28. Silversides, J. A., Perner, A. \& Malbrain, M. Liberal versus restrictive fluid therapy in critically ill patients. Intensive Care Med. 45, 1440-1442. https://doi.org/10.1007/s00134-019-05713-y (2019).

29. Jhanji, S., Lee, C., Watson, D., Hinds, C. \& Pearse, R. M. Microvascular flow and tissue oxygenation after major abdominal surgery: association with post-operative complications. Intensive Care Med. 35, 671-677. https://doi.org/10.1007/s00134-0081325-z (2009)

30. De Backer, D. et al. Microcirculatory alterations in cardiac surgery: effects of cardiopulmonary bypass and anesthesia. Ann. Thorac. Surg. 88, 1396-1403. https://doi.org/10.1016/j.athoracsur.2009.07.002 (2009).

31. Vellinga, N. A., Ince, C. \& Boerma, E. C. Microvascular dysfunction in the surgical patient. Curr. Opin. Crit. Care 16, 377-383. https://doi.org/10.1097/mcc.0b013e32833a0633 (2010).

32. De Backer, D. Microcirculatory blood flow as a new tool for perioperative fluid management. In Perioperative Fluid Management (eds Farag, E. et al.) 169-180 (Springer, 2020).

33. Koch, M. et al. Effects of propofol on human microcirculation. Br. J. Anaesth. 101, 473-478. https://doi.org/10.1093/bja/aen210 (2008).

34. Turek, Z., Sykora, R., Matejovic, M. \& Cerny, V. Anesthesia and the microcirculation. Semin. Cardiothorac. Vasc. Anesth. 13, 249-258. https://doi.org/10.1177/1089253209353134 (2009).

35. Granger, D. N. et al. Splanchnic ischaemia-reperfusion injury: mechanistic insights provided by mutant mice. Acta Physiol. Scand. 173, 83-91. https://doi.org/10.1046/j.1365-201X.2001.00888.x (2001).

36. De Backer, D., Creteur, J., Dubois, M. J., Sakr, Y. \& Vincent, J. L. Microvascular alterations in patients with acute severe heart failure and cardiogenic shock. Am. Heart J. 147, 91-99 (2004).

37. Puhl, G. et al. Initial hepatic microcirculation correlates with early graft function in human orthotopic liver transplantation. Liver Transpl. 11, 555-563. https://doi.org/10.1002/lt.20394 (2005). 
38. Schaser, K. D. et al. In vivo imaging of human pancreatic microcirculation and pancreatic tissue injury in clinical pancreas transplantation. Am. J. Transplant. 5, 341-350. https://doi.org/10.1111/j.1600-6143.2004.00663.x (2005).

39. Schmitz, V., Schaser, K. D., Olschewski, P., Neuhaus, P. \& Puhl, G. In vivo visualization of early microcirculatory changes following ischemia/reperfusion injury in human kidney transplantation. Eur. Surg. Res. 40, 19-25. https://doi.org/10.1159/00010 $7683(2008)$.

40. Rehm, M. et al. Shedding of the endothelial glycocalyx in patients undergoing major vascular surgery with global and regional ischemia. Circulation 116, 1896-1906. https://doi.org/10.1161/CIRCULATIONAHA.106.684852 (2007).

41. Donati, A. et al. A comparison among portal lactate, intramucosal sigmoid $\mathrm{Ph}$, and deltaCO2 ( $\mathrm{PaCO} 2-$ regional $\mathrm{Pco} 2)$ as indices of complications in patients undergoing abdominal aortic aneurysm surgery. Anesth. Analg. 99, 1024-1031. https://doi.org/10. 1213/01.ANE.0000132543.65095.2C (2004).

42. Nakatsuka, M. Assessment of gut mucosal perfusion and colonic tissue blood flow during abdominal aortic surgery with gastric tonometry and laser Doppler flowmetry. Vasc. Endovasc. Surg. 36, 193-198. https://doi.org/10.1177/153857440203600306 (2002).

43. Mahmood, A., Gosling, P., Barclay, R., Kilvington, F. \& Vohra, R. Splanchnic microcirculation protection by hydroxyethyl starches during abdominal aortic aneurysm surgery. Eur. J. Vasc. Endovasc. Surg. 37, 319-325. https://doi.org/10.1016/j.ejvs.2008.11.003 (2009).

44. Carden, D. L. \& Granger, D. N. Pathophysiology of ischaemia-reperfusion injury. J. Pathol. 190, 255-266. https://doi.org/10. 1002/(SICI) 1096-9896(200002)190:3\%3c255::AID-PATH526\%3e3.0.CO;2-6 (2000).

45. Haglund, U. \& Bergqvist, D. Intestinal ischemia: the basics. Langenbecks Arch. Surg. 384, 233-238 (1999).

46. Vollmar, B. \& Menger, M. D. Intestinal ischemia/reperfusion: microcirculatory pathology and functional consequences. Langenbecks Arch. Surg. 396, 13-29. https://doi.org/10.1007/s00423-010-0727-x (2011).

47. Bouattour, K., Teboul, J. L., Varin, L., Vicaut, E. \& Duranteau, J. Preload dependence is associated with reduced sublingual microcirculation during major abdominal surgery. Anesthesiology 130, 541-549. https://doi.org/10.1097/ALN.0000000000002631 (2019).

48. Whittlesey, R. H. Dynamic changes in arterial pressure pulses produced by a simulated saccular aneurysm. Am. J. Physiol. 168, 192-199. https://doi.org/10.1152/ajplegacy.1951.168.1.192 (1952).

49. Chapalain, X. et al. Association between fluid overload and SOFA score kinetics in septic shock patients: a retrospective multicenter study. J. Intensive Care 7, 42. https://doi.org/10.1186/s40560-019-0394-0 (2019).

50. Malbrain, M. et al. Intravenous fluid therapy in the perioperative and critical care setting: executive summary of the International Fluid Academy (IFA). Ann. Intensive Care 10, 64. https://doi.org/10.1186/s13613-020-00679-3 (2020).

51. Myles, P. S. et al. Restrictive versus liberal fluid therapy for major abdominal surgery. N. Engl. J. Med. 378, 2263-2274. https:// doi.org/10.1056/NEJMoa1801601 (2018).

52. Vellinga, N. A., Ince, C. \& Boerma, E. C. Elevated central venous pressure is associated with impairment of microcirculatory blood flow in sepsis: a hypothesis generating post hoc analysis. BMC Anesthesiol. 13, 17. https://doi.org/10.1186/1471-2253-1317 (2013).

53. Trepte, C. J. et al. The impact of early goal-directed fluid management on survival in an experimental model of severe acute pancreatitis. Intensive Care Med. 39, 717-726. https://doi.org/10.1007/s00134-012-2775-x (2013).

54. Jhanji, S. et al. Haemodynamic optimisation improves tissue microvascular flow and oxygenation after major surgery: a randomised controlled trial. Crit. Care 14, R151. https://doi.org/10.1186/cc9220 (2010).

55. Hiltebrand, L. B. et al. Crystalloids versus colloids for goal-directed fluid therapy in major surgery. Crit. Care 13, R40. https:// doi.org/10.1186/cc7761 (2009).

56. Loggi, S. et al. Changes in the sublingual microcirculation following aortic surgery under balanced or total intravenous anaesthesia: a prospective observational study. BMC Anesthesiol. 19, 1. https://doi.org/10.1186/s12871-018-0673-7 (2019).

57. Behem, C. R. et al. Volume based resuscitation and intestinal microcirculation after ischaemia/reperfusion injury: results of an exploratory aortic clamping study in pigs. Eur. J. Vasc. Endovasc. Surg. 57, 284-291. https://doi.org/10.1016/j.ejvs.2018.08.055 (2019).

58. Guillen, J. FELASA guidelines and recommendations. J. Am. Assoc. Lab. Anim. Sci. 51, 311-321 (2012).

59. Endo, Y. et al. Stroke volume variation (SVV) and pulse pressure variation (PPV) as indicators of fluid responsiveness in sevoflurane anesthetized mechanically ventilated euvolemic dogs. J. Vet. Med. Sci. 79, 1437-1445. https://doi.org/10.1292/jvms. 16-0287 (2017).

60. Jacques, D. et al. Pulse pressure variation and stroke volume variation during increased intra-abdominal pressure: an experimental study. Crit. Care 15, R33. https://doi.org/10.1186/cc9980 (2011).

61. da Silva Ramos, F. J. et al. Estimation of stroke volume and stroke volume changes by electrical impedance tomography. Anesth. Analg. 126, 102-110. https://doi.org/10.1213/ANE.0000000000002271 (2018).

62. Renner, J. et al. Influence of increased intra-abdominal pressure on fluid responsiveness predicted by pulse pressure variation and stroke volume variation in a porcine model. Crit. Care Med. 37, 650-658. https://doi.org/10.1097/CCM.0b013e3181959864 (2009).

63. Jung, Y. S. et al. Pulse oximeter plethysmograph variation during hemorrhage in beta-blocker-treated swine. J. Surg. Res. 256, 468-475. https://doi.org/10.1016/j.jss.2020.07.004 (2020).

64. Broch, O. et al. Dynamic and volumetric variables reliably predict fluid responsiveness in a porcine model with pleural effusion. PLoS ONE 8, e56267. https://doi.org/10.1371/journal.pone.0056267 (2013).

65. Cannesson, M. et al. Respiratory variations in pulse oximetry plethysmographic waveform amplitude to predict fluid responsiveness in the operating room. Anesthesiology 106, 1105-1111. https://doi.org/10.1097/01.anes.0000267593.72744.20 (2007).

66. Cannesson, M. et al. The ability of stroke volume variations obtained with Vigileo/FloTrac system to monitor fluid responsiveness in mechanically ventilated patients. Anesth. Analg. 108, 513-517. https://doi.org/10.1213/ane.0b013e318192a36b (2009).

67. Haas, S. et al. Prediction of volume responsiveness using pleth variability index in patients undergoing cardiac surgery after cardiopulmonary bypass. J. Anesth. 26, 696-701. https://doi.org/10.1007/s00540-012-1410-x (2012).

68. Huang, C. C. et al. Prediction of fluid responsiveness in acute respiratory distress syndrome patients ventilated with low tidal volume and high positive end-expiratory pressure. Crit. Care Med. 36, 2810-2816. https://doi.org/10.1097/CCM.0b013e3181 $86 \mathrm{~b} 74 \mathrm{e}(2008)$.

69. Fellahi, J. L. et al. A comparison of endotracheal bioimpedance cardiography and transpulmonary thermodilution in cardiac surgery patients. J. Cardiothorac. Vasc. Anesth. 26, 217-222. https://doi.org/10.1053/j.jvca.2011.06.023 (2012).

70. Fellahi, J. L. et al. Can endotracheal bioimpedance cardiography assess hemodynamic response to passive leg raising following cardiac surgery?. Ann. Intensive Care 2, 26. https://doi.org/10.1186/2110-5820-2-26 (2012).

71. Biais, M., Bernard, O., Ha, J. C., Degryse, C. \& Sztark, F. Abilities of pulse pressure variations and stroke volume variations to predict fluid responsiveness in prone position during scoliosis surgery. Br. J. Anaesth. 104, 407-413. https://doi.org/10.1093/ bja/aeq031 (2010).

72. Guinot, P. G. et al. Passive leg raising can predict fluid responsiveness in patients placed on venovenous extracorporeal membrane oxygenation. Crit. Care 15, R216. https://doi.org/10.1186/cc10451 (2011).

73. Tshomba, Y. et al. Clinical outcomes of hybrid repair for thoracoabdominal aortic aneurysms. Ann. Cardiothorac. Surg. 1, 293-303. https://doi.org/10.3978/j.issn.2225-319X.2012.07.15 (2012). 
74. Chiesa, R. et al. Use of a novel hybrid vascular graft for sutureless revascularization of the renal arteries during open thoracoabdominal aortic aneurysm repair. J. Vasc. Surg. 60, 622-630. https://doi.org/10.1016/j.jvs.2014.03.256 (2014).

75. Patel, V. I., Lancaster, R. T., Conrad, M. F. \& Cambria, R. P. Open surgical repair of thoracoabdominal aneurysms: the Massachusetts General Hospital experience. Ann. Cardiothorac. Surg. 1, 320-324. https://doi.org/10.3978/j.issn.2225-319X.2012. 09.02 (2012).

76. Boerma, E. C. \& Ince, C. The role of vasoactive agents in the resuscitation of microvascular perfusion and tissue oxygenation in critically ill patients. Intensive Care Med. 36, 2004-2018. https://doi.org/10.1007/s00134-010-1970-x (2010).

77. Stens, J. et al. Microcirculatory perfusion during different perioperative hemodynamic strategies. Microcirculation 22, 267-275. https://doi.org/10.1111/micc.12194 (2015).

78. Bansch, P., Statkevicius, S. \& Bentzer, P. Plasma volume expansion with $5 \%$ albumin compared to Ringer's acetate during normal and increased microvascular permeability in the rat. Anesthesiology 121, 817-824. https://doi.org/10.1097/ALN.0000000000 000363 (2014).

79. Kimberger, O. et al. Goal-directed colloid administration improves the microcirculation of healthy and perianastomotic colon. Anesthesiology 110, 496-504. https://doi.org/10.1097/ALN.0b013e31819841f6 (2009).

80. de Carvalho, H., Dorigo, D. \& Bouskela, E. Effects of Ringer-acetate and Ringer-dextran solutions on the microcirculation after LPS challenge: observations in the hamster cheek pouch. Shock 15, 157-162 (2001).

81. Hoffmann, J. N. et al. Hydroxyethyl starch ( $130 \mathrm{kD})$, but not crystalloid volume support, improves microcirculation during normotensive endotoxemia. Anesthesiology 97, 460-470 (2002).

82. Steinbauer, M., Harris, A. G. \& Messmer, K. Effects of dextran on microvascular ischemia-reperfusion injury in striated muscle. Am. J. Physiol. 272, H1710-1716. https://doi.org/10.1152/ajpheart.1997.272.4.H1710 (1997).

83. Schaper, J. et al. Volume therapy with colloid solutions preserves intestinal microvascular perfusion in endotoxaemia. Resuscitation 76, 120-128. https://doi.org/10.1016/j.resuscitation.2007.06.026 (2008).

84. Obonyo, N. G. et al. Effects of volume resuscitation on the microcirculation in animal models of lipopolysaccharide sepsis: a systematic review. Intensive Care Med. Exp. 4, 38. https://doi.org/10.1186/s40635-016-0112-3 (2016).

85. Kauvar, D. S., Baer, D. G., Dubick, M. A. \& Walters, T. J. Effect of fluid resuscitation on acute skeletal muscle ischemia-reperfusion injury after hemorrhagic shock in rats. J. Am. Coll. Surg. 202, 888-896. https://doi.org/10.1016/j.jamcollsurg.2006.03.003 (2006).

86. Schick, M. A. et al. Effects of crystalloids and colloids on liver and intestine microcirculation and function in cecal ligation and puncture induced septic rodents. BMC Gastroenterol. 12, 179. https://doi.org/10.1186/1471-230X-12-179 (2012).

87. Ergin, B. et al. Fully balanced fluids do not improve microvascular oxygenation, acidosis and renal function in a rat model of endotoxemia. Shock 46, 83-91. https://doi.org/10.1097/SHK.0000000000000573 (2016).

88. Guven, G., Hilty, M. P. \& Ince, C. Microcirculation: physiology, pathophysiology, and clinical application. Blood Purif. 49, 143-150. https://doi.org/10.1159/000503775 (2020).

89. Komori, M. et al. Effects of crystalloids and colloids on microcirculation, central venous oxygen saturation, and central venousto-arterial carbon dioxide gap in a rabbit model of hemorrhagic shock. J. Anesth. 33, 108-117. https://doi.org/10.1007/s00540018-2594-5 (2019).

90. Trepte, C. J. et al. Prediction of volume-responsiveness during one-lung ventilation: a comparison of static, volumetric, and dynamic parameters of cardiac preload. J. Cardiothorac. Vasc. Anesth. 27, 1094-1100. https://doi.org/10.1053/j.jvca.2013.05. 003 (2013).

91. Wodack, K. H. et al. Assessment of central hemodynamic effects of phenylephrine: an animal experiment. J. Clin. Monit. Comput. 33, 377-384. https://doi.org/10.1007/s10877-018-0204-6 (2019).

92. Haas, S. A. et al. An assessment of global end-diastolic volume and extravascular lung water index during one-lung ventilation: is transpulmonary thermodilution usable?. Anesth. Analg. 117, 83-90. https://doi.org/10.1213/ANE.0b013e31828f2c39 (2013).

93. Bachmann, K. A. et al. Effects of thoracic epidural anesthesia on survival and microcirculation in severe acute pancreatitis: a randomized experimental trial. Crit. Care 17, R281. https://doi.org/10.1186/cc13142 (2013).

94. Kluttig, R. et al. Invasive hemodynamic monitoring of aortic and pulmonary artery hemodynamics in a large animal model of ARDS. J. Vis. Exp. https://doi.org/10.3791/57405 (2018).

95. Byrne, L. et al. Unintended consequences: fluid resuscitation worsens shock in an ovine model of endotoxemia. Am. J. Respir. Crit. Care Med. 198, 1043-1054. https://doi.org/10.1164/rccm.201801-0064OC (2018).

96. Orbegozo, D. et al. Effects of different crystalloid solutions on hemodynamics, peripheral perfusion, and the microcirculation in experimental abdominal sepsis. Anesthesiology 125, 744-754. https://doi.org/10.1097/ALN.0000000000001273 (2016).

97. Chowdhury, A. H., Cox, E. F., Francis, S. T. \& Lobo, D. N. A randomized, controlled, double-blind crossover study on the effects of 2 - $\mathrm{L}$ infusions of $0.9 \%$ saline and plasma-lyte(R) 148 on renal blood flow velocity and renal cortical tissue perfusion in healthy volunteers. Ann. Surg. 256, 18-24. https://doi.org/10.1097/SLA.0b013e318256be72 (2012).

98. Funk, W. \& Baldinger, V. Microcirculatory perfusion during volume therapy. A comparative study using crystalloid or colloid in awake animals. Anesthesiology 82, 975-982. https://doi.org/10.1097/00000542-199504000-00022 (1995).

99. Zweifach, B. W. \& Lipowsky, H. H. Quantitative studies of microcirculatory structure and function. III. Microvascular hemodynamics of cat mesentery and rabbit omentum. Circ. Res. 41, 380-390. https://doi.org/10.1161/01.res.41.3.380 (1977).

100. De Backer, D., Donadello, K. \& Cortes, D. O. Monitoring the microcirculation. J. Clin. Monit. Comput. 26, 361-366. https://doi. org/10.1007/s10877-012-9383-8 (2012).

101. Kilkenny, C., Browne, W. J., Cuthill, I. C., Emerson, M. \& Altman, D. G. Improving bioscience research reporting: the ARRIVE guidelines for reporting animal research. Osteoarthr. Cartil. 20, 256-260. https://doi.org/10.1016/j.joca.2012.02.010 (2012).

102. Behem, C. R. et al. Real-time assessment of spinal cord microperfusion in a porcine model of ischemia/reperfusion. J. Vis. Exp. https://doi.org/10.3791/62047 (2020).

103. Mahjoub, Y. et al. Evaluation of pulse pressure variation validity criteria in critically ill patients: a prospective observational multicentre point-prevalence study. Br. J. Anaesth. 112, 681-685. https://doi.org/10.1093/bja/aet442 (2014).

104. Teboul, J. L., Monnet, X., Chemla, D. \& Michard, F. Arterial pulse pressure variation with mechanical ventilation. Am. J. Respir. Crit. Care Med. 199, 22-31. https://doi.org/10.1164/rccm.201801-0088CI (2019).

105. Briers, D. et al. Laser speckle contrast imaging: theoretical and practical limitations. J. Biomed. Opt. 18, 066018. https://doi.org/ 10.1117/1.JBO.18.6.066018 (2013).

106. Heeman, W., Steenbergen, W., van Dam, G. \& Boerma, E. C. Clinical applications of laser speckle contrast imaging: a review. J. Biomed. Opt. 24, 1-11. https://doi.org/10.1117/1.JBO.24.8.080901 (2019).

107. McGuire, P. G. \& Howdieshell, T. R. The importance of engraftment in flap revascularization: confirmation by laser speckle perfusion imaging. J. Surg. Res. 164, e201-212. https://doi.org/10.1016/j.jss.2010.07.059 (2010).

108. Wu, C. Y. et al. Laser speckle contrast imaging for assessing microcirculatory changes in multiple splanchnic organs and the gracilis muscle during hemorrhagic shock and fluid resuscitation. Microvasc. Res. 101, 55-61. https://doi.org/10.1016/j.mvr. 2015.06.003 (2015).

109. Bezemer, R. et al. Validation of near-infrared laser speckle imaging for assessing microvascular (re)perfusion. Microvasc. Res. 79, 139-143. https://doi.org/10.1016/j.mvr.2010.01.004 (2010).

110. Ding, C. et al. Laser speckle contrast imaging for assessment of abdominal visceral microcirculation in acute peritonitis: does sequential impairments exist?. Microvasc. Res. 95, 26-30. https://doi.org/10.1016/j.mvr.2014.06.011 (2014). 
111. Li, C. H. et al. The monitoring of microvascular liver blood flow changes during ischemia and reperfusion using laser speckle contrast imaging. Microvasc. Res. 94, 28-35. https://doi.org/10.1016/j.mvr.2014.04.010 (2014).

112. Sturesson, C., Milstein, D. M., Post, I. C., Maas, A. M. \& van Gulik, T. M. Laser speckle contrast imaging for assessment of liver microcirculation. Microvasc. Res. 87, 34-40. https://doi.org/10.1016/j.mvr.2013.01.004 (2013).

113. Senarathna, J., Rege, A., Li, N. \& Thakor, N. V. Laser speckle contrast imaging: theory, instrumentation and applications. IEEE Rev. Biomed. Eng. 6, 99-110. https://doi.org/10.1109/RBME.2013.2243140 (2013).

114. Klijn, E. et al. Laser speckle imaging identification of increases in cortical microcirculatory blood flow induced by motor activity during awake craniotomy. J. Neurosurg. 118, 280-286. https://doi.org/10.3171/2012.10.JNS1219 (2013).

115. Eriksson, S., Nilsson, J., Lindell, G. \& Sturesson, C. Laser speckle contrast imaging for intraoperative assessment of liver microcirculation: a clinical pilot study. Med. Devices (Auckl.) 7, 257-261. https://doi.org/10.2147/MDER.S63393 (2014).

116. Rousseau, P. et al. Increasing the "region of interest" and "time of interest", both reduce the variability of blood flow measurements using laser speckle contrast imaging. Microvasc. Res. 82, 88-91. https://doi.org/10.1016/j.mvr.2011.03.009 (2011).

117. Debus, E. S. et al. Feasibility study of a novel thoraco-abdominal aortic hybrid device (SPIDER-graft) in a translational pig model. Eur. J. Vasc. Endovasc. Surg. 55, 196-205. https://doi.org/10.1016/j.ejvs.2017.11.018 (2018).

118. Wipper, S. et al. Impact of hybrid thoracoabdominal aortic repair on visceral and spinal cord perfusion: the new and improved SPIDER-graft. J. Thorac. Cardiovasc. Surg. 158, 692-701. https://doi.org/10.1016/j.jtcvs.2018.11.133 (2019).

119. Youden, W. J. Index for rating diagnostic tests. Cancer 3, 32-35 (1950).

\section{Acknowledgements}

The authors would like to thank Dr. med. vet. Aline Reitmeier and Mrs. Jutta Dammann, Facility of Research Animal Care, University Medical Center Hamburg-Eppendorf, Hamburg, Germany, for providing pre- and perioperative animal care and their technical assistance on animal handling. The authors further would like to thank Dr. Daniel Manzoni, Department of Vascular Surgery, Hôpital Kirchberg, Luxembourg for his technical assistance.

\section{Author contributions}

C.R.B.: study concept and design, acquisition and interpretation of data, draft of the manuscript, statistical analysis, final approval. M.F.G.: study concept and design, acquisition and interpretation of data, draft of the manuscript, critical revision of the manuscript for important intellectual content, final approval. T.F.: acquisition and interpretation of data, critical revision of the manuscript for important intellectual content, final approval. R.K.: acquisition and interpretation of data, critical revision of the manuscript for important intellectual content, final approval. H.O.P.: interpretation of data, statistical analysis, critical revision of the manuscript for important intellectual content, final approval. A.D.: acquisition and interpretation of data, critical revision of the manuscript for important intellectual content, final approval. E.S.D.: acquisition and interpretation of data, critical revision of the manuscript for important intellectual content, final approval. D.A.R.: study concept and design, interpretation of data, critical revision of the manuscript for important intellectual content, final approval. S.H.W.: study concept and design, acquisition and interpretation of data, critical revision of the manuscript for important intellectual content, final approval. C.J.C.T.: study concept and design, acquisition and interpretation of data, critical revision of the manuscript for important intellectual content, study supervision, final approval.

\section{Funding}

Open Access funding enabled and organized by Projekt DEAL. The study was supported by departmental funds of the Department of Anesthesiology, University Medical Center Hamburg-Eppendorf, Hamburg, Germany.

\section{Competing interests}

Constantin J. C. Trepte has received honorary for lectures by Maquet. All other authors declare no competing interest.

\section{Additional information}

Supplementary Information The online version contains supplementary material available at https://doi.org/ 10.1038/s41598-021-88458-3.

Correspondence and requests for materials should be addressed to C.R.B.

Reprints and permissions information is available at www.nature.com/reprints.

Publisher's note Springer Nature remains neutral with regard to jurisdictional claims in published maps and institutional affiliations.

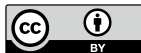

Open Access This article is licensed under a Creative Commons Attribution 4.0 International License, which permits use, sharing, adaptation, distribution and reproduction in any medium or format, as long as you give appropriate credit to the original author(s) and the source, provide a link to the Creative Commons licence, and indicate if changes were made. The images or other third party material in this article are included in the article's Creative Commons licence, unless indicated otherwise in a credit line to the material. If material is not included in the article's Creative Commons licence and your intended use is not permitted by statutory regulation or exceeds the permitted use, you will need to obtain permission directly from the copyright holder. To view a copy of this licence, visit http://creativecommons.org/licenses/by/4.0/.

(C) The Author(s) 2021 